\title{
An optimum method for latticed shells based on concept of the compression line
}

\author{
Liming Zhu, Qi Liu*, Peinan Gao, Yingyun Ma, Bingyang Guo and Wenfeng Du \\ Institute of Steel and Spatial Structures in School of Civil Engineering and Architecture, Henan University, Kaifeng \\ 475004 China \\ ${ }^{*}$ Corresponding author: dwf@henu.edu.cn
}

\begin{abstract}
To improve the load-carrying capacity latticed shells, an innovative optimum method based on the concept of compression line is proposed in this paper. The basic principle of this method is using the character without moment in compression line. The arc line which forms the contour line of sphere and latticed shell structures is substituted by compression line in this method. Then the latticed shell structures are in the state of compression, and the influence of bending stress reduces greatly. As a result, the loadcarrying capacity of the latticed shell structure is increased. Through the geometrical nonlinear analysis of a sunflower-patterned single-layer latticed shell structure with a span of $48 \mathrm{~m}$, it is found that the loadcarrying capacity of the single-layer latticed shell structure can be improved by $5.48 \%$. Furthermore, the results of 84 structural analyses of single-layer or double-layer sphere and cylinder latticed shell structures show that the optimum method is right and effective. And especially, it is applicable to single-layer latticed shell structure with rise-span ratio $1 / 5$ with the max improvement $6.4 \%$ of load-carrying capacity.
\end{abstract}

\section{Introduction}

Optimum design of the latticed shells can be divided into three levels. The first is the level of structure, which includes the macroscopical surface shape, the span, the rise, the thickness of the shells and so on. Deng Hua built a mathematical model to optimize the latticed shells to minimize the self-weight of the structure, and they discussed the accurate values for the rise and the thickness of the latticed shells [1]. The second is the level of components, which include the geometrical length of the member bar, the measurement of the grid and the design of the connecting joints. Shan Luyang set the measurement of the grid and the thickness of the latticed shell as the design variables, and the minimum of the cost as the objective function to optimize the structure [2]. The third is the level of section, which includes the optimization of the size of the section. Li Yongmei made on section optimizing under determined cable forces by means of improved optimization method of double objectives [3].

Based on the states of the research both in China and abroad, the main object of optimization is the rise-span ratio, the measurement of the grid, the thickness of the latticed shell, and the spherical or cylinder latticed shells structure are always adopted [4]-[10]. However, during the process of engineering practice, we noticed that the latticed shell structures are more likely to be in the full compressive stress states with no bending moment by substituting the arch line of the latticed shells into the compression line. From which it can increase the ultimate bearing capacity. Thus we proposed an optimum method for latticed shells based on concept of the compression line.

\section{The principle of the method}

The Peripheral hinges are used as the common constraint method of the latticed shells structure. If we take a profile of the single-layer latticed shells structure into consideration, it is easy to be found the stress characteristics of the structure are similar as two-hinged arch. As we know the selection of reasonable arch axis has a significant effect on the stress distribution and stress size of each section during the process of designing the two-hinged arch structure.

A major stress characteristic of single-layer latticed shells structure is the membrane action. Single-layer latticed shells structure has a powerful in-plane stiffness far outnumbering the out-plane stiffness. This structure uses the inplane stress to resist the vertical load and the load-carrying capacity is controlled by the buckling strength. The singlelayer latticed shell structures transmits most of the load as the axial forces of the members of latticed shells, while the sphere latticed shells structure remains some of the bending stress. Those bending stresses can sharply decrease the load-carrying capacity of the latticed shells structure. Therefore, the principle of axis selection decreases the moment created by the load as much as possible. The ideal axis is consistent with the compression line, which means all the sections in the latticed shell are under the axial compression with no bending moment and all the stress characteristics is the most reasonable at the same time. 
The load acts on the latticed shell structures can simplify to the concentrated force of each intercept point. Under the action of the vertical load along gravity direction, the compression line is usually not the circular arch. In this situation, the compression line can be confirmed by the graphic method shown in Fig.1. Firstly, calculate the support reaction $\mathrm{Rx}$ and $\mathrm{Ry}$; then assign the $\mathrm{AB}$ bar along the direction of the resultant force of $\mathrm{Rx}$ and Ry to make sure that bar $\mathrm{BC}$ has the same direction as the total force of $\mathrm{Rx}, \mathrm{Ry}$ and F1. Then the rest can be done in the same method which means the bars are assigned along the direction of total force at nodes, in another word, the direction of the member bar coincides with the compression line, which can make the internal force transmit only as the axial force in each jointing point. Thus, we can replace the arc line with the compression line in the structure of latticed shells to contain the structure in a compressive stress state and increase the loading-capacity of the latticed shells structure eventually.

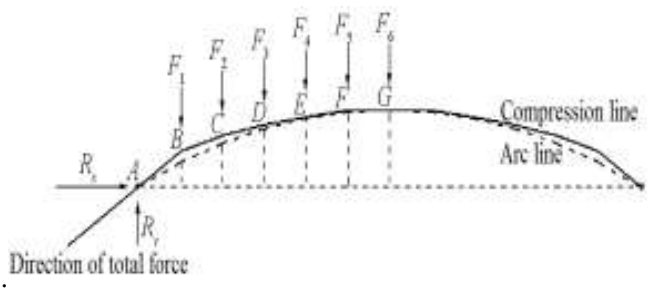

Fig. 1. Schematic compression line

\section{Analysis of examples}

In order to verify the Correctness of the optimum method, analysis is based on a sunflower-patterned single-layer latticed shell structure.

\subsection{Model of structure}

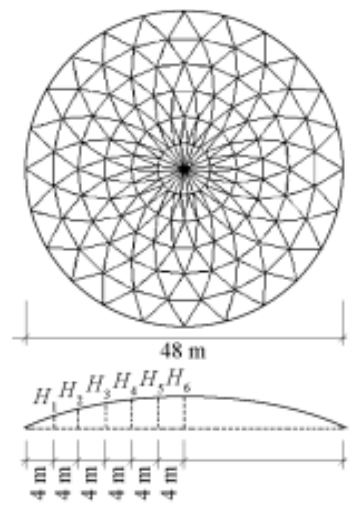

Fig. 2. Geometric model of latticed shell

Model is shown as the fig. 2 . The span of the structure is $48 \mathrm{~m}$, rise to span ratio is $1 / 6$, the total number of structure bars is 408 and nodes is 145 . Structure is clamped on supports, the steel bar is Q235 model, poison ratio is 0.3, yield strength is $215 \mathrm{~N} / \mathrm{mm}^{2}$, elastic modulus is $206 \mathrm{KN} / \mathrm{mm}^{2}$, and the cross-section of pipes we used are $133 \mathrm{~mm} \times 5 \mathrm{~mm}$. A general finite element analysis software ANSYS is used to analyse the structure and chose beam 4 as the element type of bar.

\subsection{Ideas of analysis}

At first, we set the model of the arc line, which forms the contour line of the sphere latticed shell structure. Then we set the compression line latticed shell structure model by the method indicated in fig.1. We also construct parabolic reticulated, sine line mesh and Ellipse line mesh latticed shell structure models to compare at the same time. Their difference is reflected in the data between $\mathrm{H} 1 \sim \mathrm{H} 6$, as it is presented in table 1 . We compare their ultimate carrying capacity through the geometrical nonlinear analysis.

Table 1. $H_{1} \sim H_{6}$ of different curves

\begin{tabular}{|c|c|c|c|c|c|c|}
\hline Curves & $H_{1} / \mathrm{m}$ & $H_{2} / \mathrm{m}$ & $H_{3} / \mathrm{m}$ & $H_{4} / \mathrm{m}$ & $H_{5} / \mathrm{m}$ & $H_{6} / \mathrm{m}$ \\
\hline Arc line & 2.513 & 4.523 & 6.059 & 7.142 & 7.786 & 8.000 \\
\hline Parabolic & 2.445 & 4.445 & 6.000 & 7.111 & 7.778 & 8.000 \\
\hline
\end{tabular}




\begin{tabular}{|c|c|c|c|c|c|c|}
\hline line & & & & & & \\
\hline Sine line & 2.071 & 4.000 & 5.657 & 6.928 & 7.727 & 8.000 \\
\hline Ellipse line & 4.422 & 4.422 & 6.928 & 7.543 & 7.888 & 8.000 \\
\hline $\begin{array}{c}\text { Compress- } \\
\text { ion line }\end{array}$ & 2.552 & 4.600 & 6.146 & 7.152 & 7.667 & 8.000 \\
\hline
\end{tabular}

\subsection{The result of analysis}

Fig.3 shows the result through the geometrical nonlinear analysis between five kinds of curves sunflower-patterned single-layer latticed shell structures. Ratio of current load level of design load as the load factor. We can learn curves of load displacement show highly geometrical nonlinearity from fig. 3 and the load factors of five kinds of curves for single-layer latticed shell structures have a big difference. The highest load factor belongs to compression line latticed shell structure, as a number of 14.64, then we sorts others in the order as follows, arc line mesh latticed shell structure as a number of 13.88, parabolic line reticulated latticed shell structure as a number of 13.84, sine line mesh latticed shell structure as a number of 9.09, and ellipse line mesh latticed shell structure as a number of 4.37. From the above data we know that the shape of curves has a significant impact on carrying capacity. The load carrying capacity of the compression line latticed shell is increased by $5.48 \%$ compared with the circular arc line mesh latticed shell, but ellipse line mesh latticed shell structure just play the role as $31.48 \%$ of arc line mesh latticed shell.

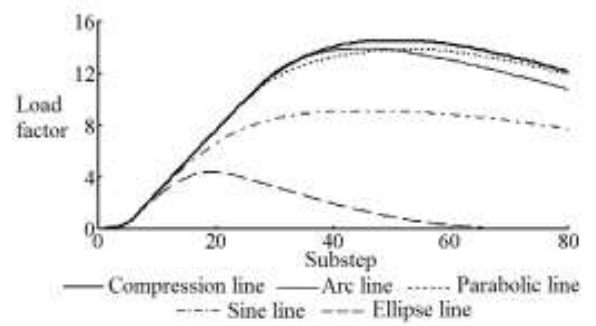

Fig. 3. Comparison of limited bearing capacity

Fig.4 shows the Nonlinear buckling geometrical model of five kinds of sunflower-patterned single-layer latticed shell structures. We can notice that the second hoop (start from the edge) of arc line mesh latticed shell structure collapsing, and inner hoop changes slightly. The feature of instability characteristics of parabolic reticulated, sine line mesh and arc line mesh latticed shell structure is nearly same. The buckling model of Ellipse line mesh latticed shell structure is the inner hoop collapsing, and outer hoop change merely. The buckling model of compression line latticed shell structure is the inner hoop has the same damage process with the outer hoop, the second hoop (start from the edge) collapsing and have large deformations at the inner hoop. We can find that arc line mesh, parabolic line reticulated and sine line mesh latticed shell structure broke down because of the instability of the outer hoop, while there are also have the potential carrying capacity at the inner hoop. The reason for Ellipse line mesh is the instability of the inner hoop, while there are also have the potential carrying capacity at the outer hoop. Due to the inner and outer hoop has the same damage process. Its potential carrying capacity plays an important role before they lose their stability. So the highest carrying capacity belongs to compression line mesh latticed shell.

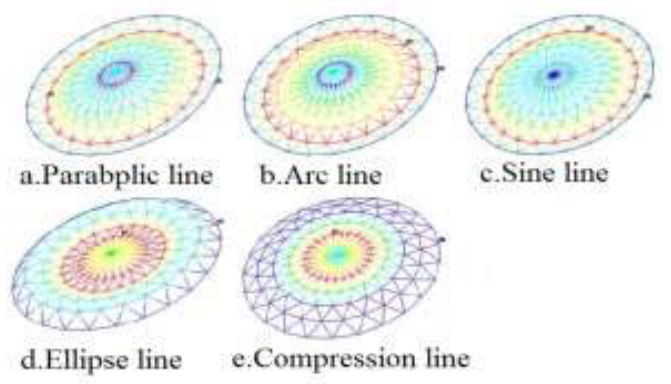

Fig. 4. Geometrical model of instability

\subsection{The result influence of the rise to span ratio}


Rise to span ratio do count much in mechanics performance of single-layer latticed shell structure. The former objects we analysed are flat reticulated latticed shell structure whose rise to span ratio are $1 / 6$, and fig.5 provides the geometrical nonlinearity results from five kinds of curving latticed shell structures whose rise to span ratio equal $1 / 10$, $1 / 8,1 / 5$, and $1 / 4$.

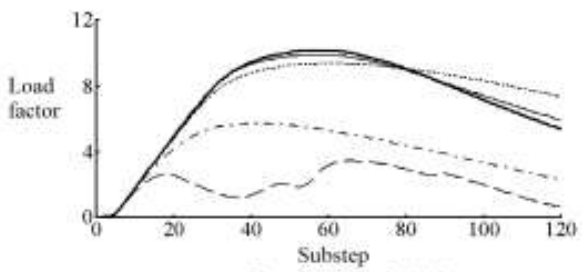

a. Rise to span ratio $1 / 10$

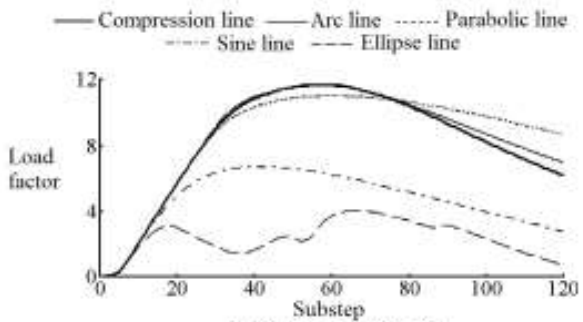

b. Rise to span ratio $1 / 8$

- Compression line _- Are line ...... Parabolic líne

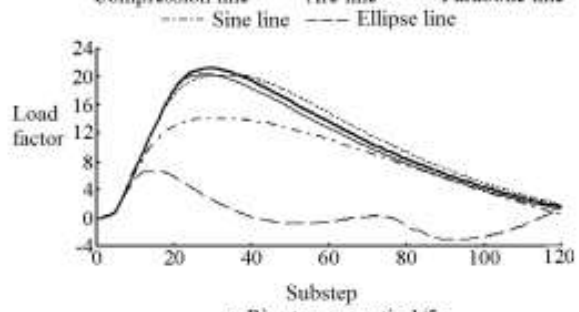

c. Rise to span ratio $1 / 5$

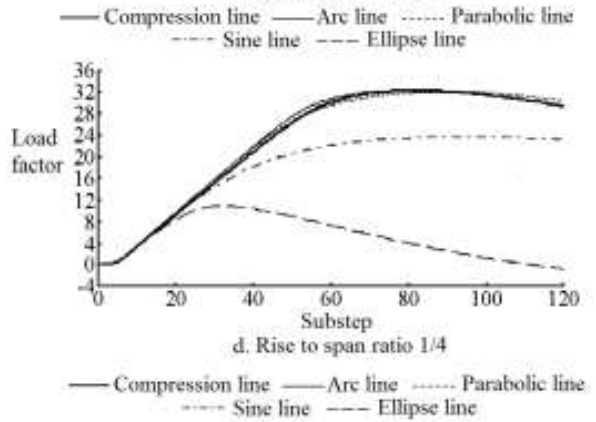

Fig. 5. Comparison of limited bearing capacity with different rise to span ratios

As we can learn from fig.5, due to vertical stiffness of the structure rising along with the number of rises to span ratio growing, the load factors of single-layer latticed shell structures increasing with. And load factor of compression line still occupied the peak. Arc line mesh, parabolic reticulated, sine line mesh, and ellipse line mesh latticed shell structure is as fallows.

We lay out $1 / 10,1 / 8,1 / 5$, and $1 / 4$ four kinds of rise to span ratios, by contrast. Load factor of compression line is improving $0.87 \%, 1.33 \%, 5.23 \%$, and $4.53 \%$ than arc wire mesh latticed shell structure. It shows that carrying capacity of the compression line rising with a rise to span ratio growing at the primary period, then declining slightly. The carrying capacity goes through the roof when rise to span ratio equals $1 / 6$. Carrying capacity increases merely when span ratio is small, but the amount of increase could be $5 \%$ when span ratio quite big.

\section{Analysis of typical structure}


To get a comprehensive understanding of the optimized effect by using this optimum method on all kinds spatial latticed shell structures, we also analysed the typical latticed shell structure which has wide application in engineering. The calculation results are presented in Table 2. Before analysing these structures, we build the model in spatial structure analysis software MATCAD firstly, then input into ANSYS to carry out geometrical nonlinear analysis.

Table 2. Optimum results of single-layer spherical latticed shell structures

\begin{tabular}{|c|c|c|c|}
\hline $\begin{array}{l}\text { Structure type } \\
\text { and diagram }\end{array}$ & $\begin{array}{l}\text { Span of } \\
\text { structure }\end{array}$ & $\begin{array}{l}\text { Rise to } \\
\text { span } \\
\text { ratio }\end{array}$ & $\begin{array}{l}\text { Carrying } \\
\text { capacity } \\
\text { increase } \\
\text { percentage }\end{array}$ \\
\hline \multirow[t]{6}{*}{ Kiewitt type } & \multirow{2}{*}{24} & $1 / 10$ & 1.8 \\
\hline & & $1 / 5$ & 4.1 \\
\hline & \multirow{2}{*}{36} & $1 / 10$ & 1.0 \\
\hline & & $1 / 5$ & 4.2 \\
\hline & \multirow{2}{*}{48} & $1 / 10$ & 1.1 \\
\hline & & $1 / 5$ & 4.5 \\
\hline \multirow{6}{*}{$\begin{array}{l}\text { Sunflower- } \\
\text { patterned }\end{array}$} & \multirow{2}{*}{24} & $1 / 10$ & 1.1 \\
\hline & & $1 / 5$ & 5.1 \\
\hline & \multirow{2}{*}{36} & $1 / 10$ & 1.3 \\
\hline & & $1 / 5$ & 5.6 \\
\hline & \multirow{2}{*}{48} & $1 / 10$ & 1.6 \\
\hline & & $1 / 5$ & 5.8 \\
\hline \multirow{6}{*}{ Geiger type } & \multirow{2}{*}{24} & $1 / 10$ & 1.3 \\
\hline & & $1 / 5$ & 4.5 \\
\hline & \multirow{2}{*}{36} & $1 / 10$ & 1.8 \\
\hline & & $1 / 5$ & 6.0 \\
\hline & \multirow{2}{*}{48} & $1 / 10$ & 2.2 \\
\hline & & $1 / 5$ & 6.4 \\
\hline \multirow{6}{*}{$\begin{array}{l}\text { Three-dimensional } \\
\text { grids type }\end{array}$} & \multirow{2}{*}{24} & $1 / 10$ & 1.3 \\
\hline & & $1 / 5$ & 5.0 \\
\hline & \multirow{2}{*}{36} & $1 / 10$ & 1.4 \\
\hline & & $1 / 5$ & 5.3 \\
\hline & \multirow{2}{*}{48} & $1 / 10$ & 1.6 \\
\hline & & $1 / 5$ & 5.7 \\
\hline \multirow{6}{*}{ Schwedler type } & \multirow{2}{*}{24} & $1 / 10$ & 1.3 \\
\hline & & $1 / 5$ & 4.6 \\
\hline & \multirow{2}{*}{36} & $1 / 10$ & 1.8 \\
\hline & & $1 / 5$ & 5.2 \\
\hline & \multirow{2}{*}{48} & $1 / 10$ & 2.0 \\
\hline & & $1 / 5$ & 5.5 \\
\hline \multirow{6}{*}{ Geodesic type } & \multirow{2}{*}{24} & $1 / 10$ & 1.6 \\
\hline & & $1 / 5$ & 5.4 \\
\hline & \multirow{2}{*}{36} & $1 / 10$ & 1.8 \\
\hline & & $1 / 5$ & 5.6 \\
\hline & \multirow{2}{*}{48} & $1 / 10$ & 2.0 \\
\hline & & $1 / 5$ & 5.8 \\
\hline
\end{tabular}

As can be observed from the Table 2, after adopting the optimum method that is proposed in this paper, the carrying capacity of six single-layer latticed shell structures can be improved. The effectiveness of improvement is approximately $10 \%$ and the ribbed dome is seen as the most obvious structure. Along with the augment of the span of various latticed shell structure, the optimized effect increased gradually. For different rise to span ratio latticed shell structures, the smaller one is a little bit better, but not significant. 
Table 3. Optimum results of single-layer cylinder latticed shell structures

\begin{tabular}{|c|c|c|c|}
\hline $\begin{array}{c}\text { Structure type and } \\
\text { diagram }\end{array}$ & $\begin{array}{c}\text { Span of } \\
\text { structure }\end{array}$ & $\begin{array}{c}\text { Rise to } \\
\text { span } \\
\text { ratio }\end{array}$ & $\begin{array}{c}\text { Carrying } \\
\text { capacity } \\
\text { increase } \\
\text { percentage }\end{array}$ \\
\hline \multirow{6}{*}{ Single-diagonal type } & \multirow{2}{*}{24} & $1 / 10$ & 1.5 \\
\hline & & $1 / 5$ & 5.3 \\
\hline & \multirow{2}{*}{36} & $1 / 10$ & 1.6 \\
\hline & & $1 / 5$ & 5.5 \\
\hline & \multirow{2}{*}{48} & $1 / 10$ & 1.9 \\
\hline & & $1 / 5$ & 5.6 \\
\hline \multirow{6}{*}{ Lamella type } & \multirow{2}{*}{24} & $1 / 10$ & 1.1 \\
\hline & & $1 / 5$ & 5.1 \\
\hline & \multirow{2}{*}{36} & $1 / 10$ & 1.4 \\
\hline & & $1 / 5$ & 5.5 \\
\hline & \multirow{2}{*}{48} & $1 / 10$ & 1.7 \\
\hline & & $1 / 5$ & 5.9 \\
\hline \multirow{6}{*}{ S\&P type } & \multirow{2}{*}{24} & $1 / 10$ & 1.5 \\
\hline & & $1 / 5$ & 5.5 \\
\hline & \multirow{2}{*}{36} & $1 / 10$ & 1.8 \\
\hline & & $1 / 5$ & 5.9 \\
\hline & \multirow{2}{*}{48} & $1 / 10$ & 1.9 \\
\hline & & $1 / 5$ & 5.6 \\
\hline \multirow{6}{*}{$\begin{array}{c}\text { Three-dimensional } \\
\text { grids type }\end{array}$} & \multirow{2}{*}{24} & $1 / 10$ & 1.2 \\
\hline & & $1 / 5$ & 5.0 \\
\hline & \multirow{2}{*}{36} & $1 / 10$ & 1.4 \\
\hline & & $1 / 5$ & 5.5 \\
\hline & \multirow{2}{*}{48} & $1 / 10$ & 1.6 \\
\hline & & $1 / 5$ & 5.7 \\
\hline \multirow{6}{*}{$\begin{array}{c}\text { Double-diagonal } \\
\text { type }\end{array}$} & \multirow{2}{*}{24} & $1 / 10$ & 1.3 \\
\hline & & $1 / 5$ & 5.2 \\
\hline & \multirow{2}{*}{36} & $1 / 10$ & 1.7 \\
\hline & & $1 / 5$ & 5.8 \\
\hline & \multirow{2}{*}{48} & $1 / 10$ & 1.5 \\
\hline & & $1 / 5$ & 6.0 \\
\hline \multirow{6}{*}{ "mi" type } & \multirow{2}{*}{24} & $1 / 10$ & 1.6 \\
\hline & & $1 / 5$ & 5.4 \\
\hline & \multirow{2}{*}{36} & $1 / 10$ & 1.8 \\
\hline & & $1 / 5$ & 5.6 \\
\hline & \multirow{2}{*}{48} & $1 / 1$ & 2.1 \\
\hline & & $1 / 5$ & 6.1 \\
\hline
\end{tabular}

Table 3 provides optimum calculation results of six single-layer cylinder latticed shell structures such as singlediagonal type, lamella type, S\&P type, three-dimensional grids type, double-diagonal type, and "mi" type. As indicated in table 3, after adopting the optimum method proposed in the paper, the carrying capacity of six single-layer cylinder latticed shell improved too. The maximal improvement effect can reach about $6.4 \%$, and union square type is the most obvious one. With the augment of span, the optimum effect increased a little. And the rule that a smaller rise-span ratio is fractional better than the bigger is the same as the rule of single-layer spherical shell structure.

The optimum results of double-layer latticed shell structure are shown in Table 4. Quadrangular pyramid system sphere and cylinder latticed shell structure which are frequently used were chose in the calculation. From the Table 4, the range of the carrying capacity of double-layer shell structure rarely improved after using the optimum method. The maximal effect of improvement is lower than $3 \%$.

Table 4. Optimum results of double-layer latticed shell structures

\begin{tabular}{|c|c|c|c|}
\hline $\begin{array}{c}\text { Structure type and } \\
\text { diagram }\end{array}$ & $\begin{array}{l}\text { Span of } \\
\text { structure }\end{array}$ & $\begin{array}{c}\text { Rise to } \\
\text { span } \\
\text { ratio }\end{array}$ & $\begin{array}{l}\text { Carrying } \\
\text { capacity } \\
\text { increase } \\
\text { percentage }\end{array}$ \\
\hline \multirow{6}{*}{ 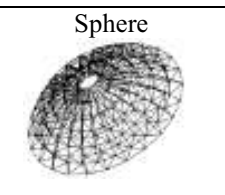 } & \multirow{2}{*}{36} & $1 / 10$ & 0.7 \\
\hline & & $1 / 5$ & 1.1 \\
\hline & \multirow{2}{*}{72} & $1 / 10$ & 0.9 \\
\hline & & $1 / 5$ & 1.3 \\
\hline & \multirow{2}{*}{96} & $1 / 10$ & 0.2 \\
\hline & & $1 / 5$ & 2.0 \\
\hline \multirow[t]{2}{*}{ Cylinder } & \multirow{2}{*}{36} & $1 / 10$ & 1.3 \\
\hline & & $1 / 5$ & 1.5 \\
\hline
\end{tabular}




\begin{tabular}{|c|c|c|c|}
\hline \multirow{2}{*}{ 3. } & \multirow{2}{*}{72} & $1 / 10$ & 1.4 \\
\cline { 3 - 4 } & & $1 / 5$ & 1.4 \\
\cline { 3 - 4 } & \multirow{2}{*}{26} & $1 / 10$ & 1.8 \\
\cline { 3 - 4 } & & $1 / 5$ & 2.2 \\
\hline
\end{tabular}

\section{Conclusions}

1) Through the example analysis of the sunflower-patterned single-layer latticed shell structure with a span of $48 \mathrm{~m}$, it proved that the ultimate carrying capacity of the compression line latticed shell is the highest. The optimum method for latticed shells based on concept of compression line proposed in this paper is quite true.

2) Compared with the figures of $H_{1} \sim H_{6}$ of different curve forms, we can know that the dimension of several singlelayer latticed shell structure is comparable with each other, but the carrying capacity reflects notable differences. From another perspective, this also can be an evidence to prove the single-layer latticed shell structure is defect-sensitive structure.

3) Summarizing 84calculation results of single-layer spherical latticed shell, single-layer cylinder latticed shell, double-layer spherical latticed shell and double-layer cylinder latticed shell. It is knowable that an optimum method for latticed shells based on concept of compression line has good effect on single-layer latticed shells with a rise-span ratio at about $1 / 5$ and the ultimate carrying capacity can improve $6.4 \%$. Yet the effect on double-layer latticed shells and single-layer latticed shells with rise-span ratio at about $1 / 10$ is considered to be insignificant.

4) Carrying coefficients of latticed shells varies greatly when using different geometric curve types. The carrying capacity of the compression line, arch line and parabolic line is quite high. And the capacity of ellipse line is relatively low, only about $1 / 3$ of arc line mesh latticed shell.

\section{References}

1. Hua D, Shilin D, "Shape optimization of spatial latticed shell structures" Journal of Zhejiang University(Engineering Science), 4,371, (1999)

2. Luyang S, Hui Y, "Optimization analysis of large-span double-layer cylindrical latticed shell structure" Journal of Building Structures, 6,47, ( 1999)

3. Yongmei L, Yigang Z, Xuesong L, "Section optimization of cable-supported latticed shell" Industrial Construction, 4,73, (2007)

4. Zongfa L, Zhitao Y, Yansheng W, Zuoliang L, "Scheme selection and optimum design of single-layer reticulated domes" Journal of Civil,Architectural \& Environmental Engineering, 4, 83, ( 2007)

5. Yueqin Q, "Optimization design of large-span spatial latticed” Construction Technology, 2,393, ( 2009)

6. Kashani M, Croll J G A, "Non-linear buckling response of spherical space-domes" Progress in shell and spatial structures, IASS,(1989)

7. Chai S, Sun H C, “A relative difference quotient algorithm for discrete optimization" Structural Optimization, 1, 46,(1966)

8. Dong W, Jing L, "Shape optimization of space trusses subject to frequency constralnts" Engineering Mechanics, 4, $22,(2007)$

9. Salajegheh E, Vanderplaats G N, "Optimum design of trusses with sizing and shape variables" Structural Optimization, 6,79, (1993)

10. Rajah S D, "Sizing, shape, and topology design optimization of trusses using genetic algorithm" Journal of Structural Engineering, 10, 1480, (1995) 